OPEN ACCESS

Edited by:

Namrita Lall,

University of Pretoria, South Africa

Reviewed by:

Sefirin Dijogue,

University of Yaounde I, Cameroon

SubbaRao V. Madhunapantula, JSS Academy of Higher Education

and Research, India

${ }^{*}$ Correspondence:

Young Ho Kim

yhk@cnu.ac.k

Young Ran Kim

kimyr@jnu.ac.kr

${ }^{\text {t}}$ These authors have contributed equally to this work

Specialty section: This article was submitted to Ethnopharmacology, a section of the journal

Frontiers in Pharmacology

Received: 13 October 2019

Accepted: 31 March 2020

Published: 23 April 2020

Citation:

Park JU, Yang SY, Guo RH, Li HX,

Kim YH and Kim YR (2020) AntiMelanogenic Effect of Dendropanax morbiferus and Its Active Components via Protein Kinase A/Cyclic Adenosine Monophosphate-Responsive Binding Protein- and p38 Mitogen-Activated

Protein Kinase-Mediated

Microphthalmia-Associated Transcription Factor Downregulation.

Front. Pharmacol. 11:507.

doi: 10.3389/fphar.2020.00507

\section{Anti-Melanogenic Effect of} Dendropanax morbiferus and Its Active Components via Protein Kinase A/Cyclic Adenosine Monophosphate-Responsive Binding Protein- and p38 Mitogen-Activated Protein Kinase-Mediated Microphthalmia-Associated Transcription Factor Downregulation

\author{
Jung Up Park ${ }^{1 \dagger}$, Seo Young Yang ${ }^{2 \dagger}$, Rui Hong Guo ${ }^{1}$, Hong Xu $\mathrm{Li}^{2}$, Young Ho Kim ${ }^{2 *}$ \\ and Young Ran $\mathrm{Kim}^{1 *}$ \\ ${ }^{1}$ College of Pharmacy and Research Institute of Drug Development, Chonnam National University, Gwangju, South Korea,
${ }^{2}$ College of Pharmacy, Chungnam National University, Daejeon, South Korea
}

Dendropanax morbiferus H. Lév has been reported to have some pharmacologic activities and also interested in functional cosmetics. We found that the water extract of $D$. morbiferus leaves significantly inhibited tyrosinase activity and melanin formation in $\alpha$ melanocyte stimulating hormone (MSH)-induced B16-F10 cells. D. morbiferus reduced melanogenesis-related protein levels, such as microphthalmia-associated transcription factor (MITF), TRP-1, and TRP-2, without any cytotoxicity. Two active ingredients of $D$. morbiferus, (10E)-9,16-dihydroxyoctadeca-10,17-dien-12,14-diynoate (DMW-1) and (10E)-(-)-10,17-octadecadiene-12,14-diyne-1,9,16-triol (DMW-2) were identified by testing the anti-melanogenic effects and then by liquid chromatography-tandem mass spectrometry (LC/MS/MS) analysis. DMW-1 and DMW-2 significantly inhibited melanogenesis by the suppression of protein kinase A (PKA)/cyclic AMP (cAMP)responsive binding protein (CREB) and p38 MAPK phosphorylation. DMW-1 showed a better inhibitory effect than DMW-2 in $\alpha-M S H$-induced B16-F10 cells. D. morbiferus and its active component DMW-1 inhibited melanogenesis through the downregulation of cAMP, p-PKA/CREB, p-p38, MITF, TRP-1, TRP-2, and tyrosinase. These results indicate that $D$. morbiferus and DMW-1 may be useful ingredients for cosmetics and therapeutic agents for skin hyperpigmentation disorders.

Keywords: Dendropanax morbiferus, (10E)-9,16-dihydroxyoctadeca-10,17-dien-12,14-diynoate, antimelanogenesis, tyrosinase, PKA/CREB, p-p38/p38, microphthalmia-associated transcription factor 


\section{INTRODUCTION}

Dendropanax morbiferus H. Lév, an endemic species in South Korea, has been used as an alternative traditional medicine for several diseases, such as headache, dysmenorrhea, infectious disorders, and skin disorders, for a long time (Jeong et al., 1995; Park et al., 2004; Lim et al., 2015). There are some reports that $D$. morbiferus shows anti-hyperuricemic, antiamnesic, anti-obesity, immunomodulatory, and antiinflammatory activities (Birhanu et al., 2018; Cho et al., 2018; Park et al., 2018a; Park et al., 2018b; Song et al., 2018; Sun et al., 2018b; Choo et al., 2019). The corresponding ingredients isolated from $D$. morbiferus leaves, such as 1-tetradecanol, dendropanoxide, rutin, gentisic acid, or oleifoliosides, have been reported to have diverse therapeutic potentials (Yu et al., 2012; Lee et al., 2013; Park et al., 2014; Park et al., 2017; Sun et al., 2018a). Recently, D. morbiferus is increasingly interested in functional materials in cosmetics. Several studies have reported that $D$. morbiferus and its components have diverse therapeutic potentials, such as anti-wrinkle, hair growth, and moisturizing effect (Lee et al., 2005; Deng et al., 2010; Lee et al., 2015; Park and Han, 2016). In addition, 1 -tetradecanol, and $\beta$-sitosterol isolated from $D$. morbiferus were reported to possess anti-melanogenic effects (Lee et al., 2015). However, there are few studies about the anti-melanogenic activity of D. morbiferus and its components as a therapeutic potential herbal medicine. To further investigate the efficacy and mechanism of D. morbiferus, we evaluated its function on the anti-melanogenic effect in this study.

Melanin is produced from melanocytes in the epidermis by a pigmentation process called melanogenesis and it is the main factor determining the color of human skin, hair, and eyes (Riley, 2003; D'Orazio et al., 2013). In addition, melanin plays an important role in protecting the skin from ultraviolet (UV) radiation (Costin and Hearing, 2007; Brenner and Hearing, 2008). Moreover, abnormal accumulation of melanin on skin surface leads to various skin pigmentation disorders, such as dots, freckles, and post-inflammatory hyperpigmentation (Pillaiyar et al., 2018). Melanogenesis regulation is under a complex control. Tyrosinase, the main melanogenic factor in the biosynthesis of melanin, is a rate-limiting enzyme that catalyzes the hydroxylation of L-tyrosine to form tyrosine to 3,4-dihydroxyphenylalanine (DOPA) and oxidizes DOPA to produce DOPA-quinone (Slominski et al., 1988; Slominski et al., 2012). The main role of the melanin-synthesizing tyrosinase gene family (tyrosinase, TRP-1, and TRP-2) is to differentiate, proliferate, and accumulate melanin into melanocytes. Microphthalmia-associated transcription factor (MITF) is not only the main regulator of melanocyte proliferation, development, and survival but also a major

Abbreviations: ACTH, adrenocorticotropic hormone; cAMP, cyclic adenosine monophosphate; CC, column chromatography; CREB, cAMP-responsive binding protein; DOPA, 3,4-dihydroxyphenylalanine; ERK, extracellular signal-regulated kinase $1 / 2$; JNK, c-Jun $\mathrm{N}$ terminal kinase $1 / 2$; MAPK, mitogen-activated protein kinase; MC1R, melanocortin type 1 receptor; MITF, microphthalmia-associated transcription factor; PKA, protein kinase A; SCF, stem cell factor; TRP-1, tyrosinase-related protein-1; TRP-2, tyrosinase-related protein-2; UV, ultraviolet; $\alpha$-MSH, $\alpha$-melanocyte stimulating hormone. regulator of melanogenesis-related protein expression (Liu and Fisher, 2010; Kim et al., 2018b). cAMP-responsive binding protein (CREB) regulates the expression of MITF in melanosomes. The phosphorylation of CREB is regulated by the activation of cAMP/protein kinase A (PKA) cascades that are known to play main roles in melanin synthesis (Pillaiyar et al., 2017). The mitogen-activated protein kinase (MAPK) family proteins, including extracellular signal-regulated kinase (ERK) $1 / 2$, c-Jun $\mathrm{N}$ terminal kinase (JNK) $1 / 2$ and p38, are known to play crucial roles in melanogenesis (Wu et al., 2011; Pillaiyar et al., 2017). Melanin production can be triggered by a variety of factors, including $\alpha$-melanocyte stimulating hormone ( $\alpha$-MSH), adrenocorticotropic hormone (ACTH), and stem cell factor (SCF) (Chan et al., 2014). Specifically, $\alpha-\mathrm{MSH}$ is a key regulator in the biosynthesis of melanin and prompt expression of cAMP.

Here, we found that $D$. morbiferus inhibited tyrosinase activity and melanin formation in melanocytes. In the present study, we have investigated the molecular mechanisms of $D$. morbiferus on anti-melanogenic effects by testing MITF, TRP-1, TRP-2, cAMP, PKA/CREB, and p38 MAPK pathways. In addition, the active ingredients from $D$. morbiferus were identified by LC/MS/MS analysis.

\section{MATERIAL AND METHODS}

\section{Chemical and Reagents}

L-3,4-dihydroxyphenylalanine (L-DOPA), $\alpha$-melanocyte stimulating hormone $(\alpha-\mathrm{MSH})$, and arbutin were purchased from Sigma-Aldrich Chemical Co. (St. Louis, MO, USA). Dulbecco's modified Eagle's medium (DMEM) was obtained from Welgene (DG, KR). Fetal bovine serum (FBS) and penicillin/streptomycin were purchased from Thermo Fisher Scientific (MA, USA). Lysis buffer and 3-(4, 5-dimethylthiazol2-yl)-5-(3 carboxymethoxyphenyl)-2-(4-sulfophenyl)-2Htetrazolium (MTS) were purchased from Promega (WI, USA). Antibodies specific to MITF, tyrosinase, TRP-1, TRP-2, p38, and GAPDH were purchased from Santa Cruz Biotechnology (CA, USA). Antibodies specific to PKA, CREB, ERK, JNK, p-CREB, pPKA, p-p38, p-ERK, and p-JNK proteins were obtained from Cell Signaling Technology (MA, USA). Horseradish peroxidaseconjugated secondary antibodies were purchased from Jackson ImmunoResearch, Inc. (PA, USA). Western Bright ${ }^{\mathrm{TM}} \mathrm{ECL}$ reagent was purchased from Advansta, Inc. (CA, USA). All other chemicals were used in analytical reagent grade.

\section{Preparation of the Water Extract From $D$. morbiferus Leaves and Its Fractions}

D. morbiferus water extract (W-DP) and its ethyl acetate fraction (W-EA) were prepared using a method described previously (Park et al., 2018a). D. morbiferus leaves were obtained from Jangheung (JL, KR) and the origin of the herbal medicine was confirmed by Jeollanam-do institute of natural resources research (JL, KR). D. morbiferus leaves were extracted once with water at $100^{\circ} \mathrm{C}$ for $4 \mathrm{~h}$. The filtered extract was 
concentrated using a continuous vacuum evaporator $\left(40^{\circ} \mathrm{C}, 670\right.$ $\mathrm{mmHg}$ ) followed by lyophilization in a vacuum drier (770 $\mathrm{mmHg}$ ). The crude water extract of $D$. morbiferus leaves (WDP, $20 \mathrm{~g}$ ) was suspended in water and successively divided with 3 $\mathrm{x} 1 \mathrm{~L}$ volumes of $\mathrm{n}$-hexane, chloroform, ethyl acetate, $\mathrm{n}$-butanol, and aqueous fraction, respectively. The fractions were concentrated using a rotary vacuum concentrator and dried in a $50^{\circ} \mathrm{C}$ dry oven for $>48 \mathrm{~h}$ as described previously (Park et al., 2018a).

\section{Cell Cultures and Cell Viability Assay}

Murine B16-F10 skin melanoma cells supplied by the Korea Cell Line Bank (SEL, KR) were maintained in DMEM supplemented with $1 \%$ penicillin/streptomycin and $10 \%$ FBS under an atmosphere of $5 \% \mathrm{CO}_{2}$ in a humidified $37^{\circ} \mathrm{C}$ incubator. To test cell viability, B16-F10 cells were cultured into 96-well plates (SPL Life Sciences Co., GG, KR) at a density of $0.5 \times 10^{4}$ cells/well overnight. The cells were treated with $\mathrm{W}-\mathrm{DP}$ at different concentrations with or without $\alpha-\mathrm{MSH}(200 \mathrm{nM})$ for an additional $72 \mathrm{~h}$. The viability of B16-F10 cells was determined by MTS according to the manufacturer's instructions, and absorbance was read at $490 \mathrm{~nm}$ with an ELx808 ELISA microplate reader (BioTek Instruments, Inc., VT, USA).

\section{Intracellular Tyrosinase Activity}

Intracellular tyrosinase activity was tested according to previously described method (Li et al., 2018). B16-F10 cells (1 X $10^{5}$ cells/well) were treated with W-DP $(0.03,0.1,0.3,0.5 \mathrm{mg} /$ $\mathrm{ml})$, W-EA $(0.01,0.03 \mathrm{mg} / \mathrm{ml})$, or arbutin $(0.54 \mathrm{mg} / \mathrm{ml})$ for $2 \mathrm{~h}$, followed by addition with $\alpha$-MSH (200 nM) for $72 \mathrm{~h}$. The cells were then lysed in a lysis buffer with protease inhibitor cocktail for $30 \mathrm{~min}$ at $4^{\circ} \mathrm{C}$ and centrifuged at $13,000 \mathrm{rpm}$ for $10 \mathrm{~min}$ at $4^{\circ}$ C. Proteins were quantified by using Bradford reagent and equal amounts of the lysates were treated with L-DOPA $(2 \mathrm{mg} / \mathrm{ml})$ in 96-well plates at $37^{\circ} \mathrm{C}$ for $2 \mathrm{~h}$. The production of DOPAchrome was measured using an ELISA microplate reader at an absorbance of $490 \mathrm{~nm}$.

\section{Melanin Content Assay}

B16-F10 cells $\left(1 \times 10^{5}\right.$ cells/well) were incubated into six-well plate (SPL Life Sciences Co., GG, KR) at $37^{\circ} \mathrm{C}$ overnight. B16-F10 cells were pretreated with W-DP $(0.03,0.1,0.3 \mathrm{mg} / \mathrm{ml}), \mathrm{W}$-EA $(0.01 \mathrm{mg} / \mathrm{ml})$, or arbutin $(0.54 \mathrm{mg} / \mathrm{ml})$ for $2 \mathrm{~h}$ prior to stimulation with $\alpha-\mathrm{MSH}(200 \mathrm{nM})$ for $72 \mathrm{~h}$. The cells were dissolved in $1 \mathrm{~N} \mathrm{NaOH}$ with $10 \%$ DMSO for $1 \mathrm{~h}$ at $60^{\circ} \mathrm{C}$. Total melanin lysates were transferred to a 96-well plate and the optical absorbance was measured at $405 \mathrm{~nm}$ using an ELISA microplate reader.

\section{Preparation of Active Compounds From $D$. morbiferus}

Dried leaves and stems of Dendropanax morbiferus H. Lév were purchased from the herbal company Hanna Arboretum, Goheung, Jeollanam-do, Korea and taxonomically identified by one of the authors (Prof. YHK). A voucher specimen (CNU 17002) was deposited at the Herbarium of the College of
Pharmacy, Chungnam National University. D. morbiferus (3.0 $\mathrm{kg}$ ) were extracted with 10-fold volume of boiling methanol (10 L $\times 3$ ) under reflux condition for three times. The methanol extract (470.0 g) was suspended in water and partitioned with $n$-hexane, $\mathrm{CH}_{2} \mathrm{Cl}_{2}$, EtOAc, and $n$-BuOH to yield $n$-hexane fraction, $\mathrm{CH}_{2} \mathrm{Cl}_{2}$ fraction, EtOAc fraction, $n-\mathrm{BuOH}$ fraction, and aqueous fraction, respectively.

Column chromatography (CC) separations were performed using silica gels $\left(\mathrm{SiO}_{2} ; 70-230,230-400 \mu \mathrm{m}\right.$ particle size; Fuji Silysia Chemical Ltd., AIC, JP) and $\mathrm{C}_{18}$ resins (75 $\mu \mathrm{m}$, Fuji Silysia Chemical Ltd., AIC, JP). Thin-layer chromatography (TLC) separations were performed using pre-coated silica gels 60 F254 and reversed-phase F254S plates (Merck, Darmstadt, Germany). The NMR spectra were recorded using a JEOL ECA 600 spectrometer $(1 \mathrm{H}, 600 \mathrm{MHz} ; 13 \mathrm{C}, 150 \mathrm{MHz}$; JEOL Ltd, TKY, JP). High-resolution electrospray ionization mass spectra (HRESIMS) were obtained using an Agilent 6530 accurate-mass quadrupole-time of flight-liquid chromatography/mass spectrometry (Q-TOF LC/MS).

The EtOAc extract (21.8 g) was subjected to a silica gel column chromatography with a gradient of $\mathrm{CHCl}_{3}-\mathrm{MeOH}$ $(60: 1-0: 1)$ to give ten fractions (Fr. EA1-EA10). Fraction EA3 (900.0 mg) was chromatographed on a silica gel column using a gradient of n-hexane-EtOAc (6:1-3:1) elution solvent to give eight subfractions (EA3A-EA3H), then subfraction EA3C (70.0 $\mathrm{mg}$ ) was chromatographed on a reverse-phase column with a gradient of $\mathrm{MeOH}$-water (9:1-1:0) to obtain three subfractions (EA3C1-EA3C3), subfraction EA3C1 (10.0 mg) was further chromatographed on a reverse-phase column with $\mathrm{MeOH}-$ water (3:1) to yield DMW-1 (5.3 mg). Subfraction EA3D (238.0 mg) was chromatographed on a reverse-phase column with a gradient of $\mathrm{MeOH}-$ water (3:7-1:0) to give eleven subfractions (EA3D1-EA3D11), then subfraction EA3D9 (13.6 $\mathrm{mg}$ ) was further purified by a silica gel column chromatography with $n$-hexane-acetone (1:1) to yield DMW-2 (6.2 mg). (10E)9,16-dihydroxyoctadeca-10,17-dien-12,14-diynoate (DMW-1): $\mathrm{C}_{19} \mathrm{H}_{26} \mathrm{O}_{4}$; viscous liquid; $[\alpha]-38.0^{\circ}$ ( $\left.\mathrm{c}=0.1, \mathrm{MeOH}\right)$; HRESIMS ([M+NH4]+ m/z 336.2167, calcd for 336.2175); 1DNMR (CD3OD, $600 \mathrm{MHz}$ ), and 2D-NMR data (CD3OD, 600 $\mathrm{MHz}$ ) (see Supplementary Figure S2 and Table S1). (10E)(-)-10,17-Octadecadiene-12,14-diyne-1,9,16-triol (DMW-2): $\mathrm{C}_{18} \mathrm{H}_{26} \mathrm{O}_{3}$; viscous liquid; $[\alpha]-32.6^{\circ}$ (c = 0.1, $\left.\mathrm{MeOH}\right)$; HRESIMS $([\mathrm{M}+\mathrm{NH} 4]+\mathrm{m} / \mathrm{z}$ : 308.2219 , calcd for 308.2226); 1D-NMR (CD3OD, $600 \mathrm{MHz}$ ), and 2D-NMR data (CD3OD, $600 \mathrm{MHz}$ ) (see Supplementary Figure S3 and Table S1). Stock solutions of samples were diluted using phosphate buffered saline (PBS) or ethanol and filter-sterilized and then diluted with PBS for the working concentrations.

\section{Liquid Chromatography-Tandem Mass Spectrometry (LC/MS/MS) Analysis}

Methanol (Sigma-Aldrich Chemical Co., MO, USA) and formic acid (Kanto, Chemical, TKY, JP) were used as LC/MS/MS gradient solvents. A Shimadzu HPLC system (KT, JP) consisted of a dual solvent pump LC-20A, an autosampler SIL$20 \mathrm{AC}$, and a column oven Gemini $\mathrm{C}_{18}$. In addition, a mass 
spectrometer Q-Trap 4000 (AB Sciex, Foster City, CA) was used for the separation and detection of the single compounds. In detail, the separation was carried out on a Gemini $\mathrm{C}_{18}$ column (CA, USA) $(5 \mu \mathrm{m}, 50 \mathrm{~mm} \times 2.0 \mathrm{~mm})$. The column was heated to $40^{\circ} \mathrm{C}$ under a flow rate of $0.3 \mathrm{ml} / \mathrm{min}$ with a stepwise elution from mobile phase A $(0.1 \%$ formic acid in water) to mobile phase B $(0.1 \%$ formic acid in acetonitrile) as follows. The linear gradient elution was set at $15-60 \% \mathrm{~B}$ for $1-4 \mathrm{~min}, 60 \% \mathrm{~B}$ for $5 \mathrm{~min}$, and $15 \%$ B for $10-12 \mathrm{~min}$. The injection volume was set at $10 \mu \mathrm{l}$. The mass spectrometer was operated in positive Turbo Ion Spray mode and multiple reaction monitoring (MRM) scan mode. The instrumental mass parameters were set as follows: ion spray voltage $5500 \mathrm{~V}$, curtain gas 20, GS1 60, GS2 60, and desolvation temperature (TEM) $500^{\circ} \mathrm{C}$. First, we identified the full scan of mass spectra and product ion mass spectra of DMW-1 and 2. The mass transition of $\mathrm{m} / \mathrm{z} 301.212$ to $\mathrm{m} / \mathrm{z} 55.2$ was used for DMW-1, and m/z 273.213 to m/z 55.1 was used for DMW-2. AB Sciex Analyst software (version 1.5.2) was used for data integration.

The stock solutions of DMW-1 and 2 were prepared at a concentration of $100 \mu \mathrm{M}$ in methanol. The linear range of the calibration curve for DMW-1 and 2 was diluted in water at concentrations of $5,20,50,200$, and $500 \mathrm{ng} / \mathrm{ml}$ and used as a working standard. The stock solution of $\mathrm{W}$-DP was prepared in water at concentrations of 10 and $50 \mathrm{mg} / \mathrm{ml}$. The solutions were stored at $4^{\circ} \mathrm{C}$.

\section{Cyclic AMP Assay}

B16-F10 cells were cultured at a density of $5 \times 10^{4}$ cells/well in 24-well plates for $24 \mathrm{~h}$ and pretreated with W-DP $(0.1 \mathrm{mg} / \mathrm{ml})$, W-EA $(0.01 \mathrm{mg} / \mathrm{ml})$, DMW-1 $(10 \mu \mathrm{M})$, or arbutin $(0.54 \mathrm{mg} / \mathrm{ml})$ for $2 \mathrm{~h}$, followed by treatment with $\alpha$-MSH (200 nM) for $24 \mathrm{~h}$. The cells were incubated with cell lysis buffer at $-80^{\circ} \mathrm{C}$ overnight, and then, the thawed lysates were harvested and centrifuged at $10,000 \mathrm{rpm}$ for $10 \mathrm{~min}$ at $4^{\circ} \mathrm{C}$. The cAMP levels in the lysates were measured by ELISA following the manufacturer's experimental protocols (R\&D Systems, MN, USA).

\section{Western Blot Analysis}

The target proteins were detected by Western blot analysis with specific antibodies. B16-F10 cells were cultured in 6-well plates at a density of $1.5 \times 10^{5}$ cells/well and then incubated overnight. The cells were pretreated with W-DP $(0.1 \mathrm{mg} / \mathrm{ml}), \mathrm{W}$-EA $(0.01$ $\mathrm{mg} / \mathrm{ml}), \mathrm{DMW}-1(10 \mu \mathrm{M}), \mathrm{DMW}-2(10 \mu \mathrm{M})$, or arbutin $(0.54$ $\mathrm{mg} / \mathrm{ml})$ for $2 \mathrm{~h}$ before stimulation with $\alpha-\mathrm{MSH}(200 \mathrm{nM})$ for 24 h. Then, cell lysates were harvested and centrifuged at 13,000 $\mathrm{rpm}$ for $10 \mathrm{~min}$ at $4^{\circ} \mathrm{C}$. Equal amounts of the lysates were electrophoresed on $10 \%$ sodium dodecyl sulfate-polyacrylamide gel electrophoresis (SDS-PAGE) and transferred to polyvinylidene difluoride membranes (PVDF) (Millipore, MA, USA) at $400 \mathrm{~mA}$ for $2 \mathrm{~h}$. The membranes were incubated with appropriate primary antibodies against MITF, tyrosinase, TRP-1, TRP-2, PKA, CREB, ERK, JNK, p-38, p-PKA, p-CREB, p-ERK, p-JNK, p-p38, or GAPDH at $4^{\circ} \mathrm{C}$ overnight. After being washed, the membranes were incubated with horseradish peroxidaseconjugated anti-rabbit or anti-mouse immunoglobulin secondary antibodies for $1 \mathrm{~h}$. Immunoreactive proteins were conducted using Western Bright ${ }^{\mathrm{TM}}$ ECL reagents and a C300 chemiluminescence imager (Azure Biosystems, Inc., CA, USA).

\section{Statistical Analysis}

All experiments were repeated in at least three separate experiments. Statistical differences were evaluated using GraphPad Prism version 5.01 (San Diego, CA, USA). The results shown are representative experiments. We used one-way ANOVA for multi-group comparisons followed by a Tukey post hoc test; $p<0.05$ was considered statistically significant.

\section{RESULTS}

\section{Effect of W-DP on Cell Viability, Tyrosinase Activity and Melanin Formation in $\alpha-\mathrm{MSH}-$ Induced B16-F10 Cells}

We studied the effect of W-DP on melanogenesis in B16-F10 melanoma cells. First, the cell viability was tested after treatment with various concentrations of W-DP in the presence or absence of $\alpha$-MSH. W-DP showed no cytotoxicity to B16-F10 cells (Figure 1A). Next, W-DP $(0.03,0.1,0.3,0.5 \mathrm{mg} / \mathrm{ml})$ significantly reduced intracellular tyrosinase activity and melanin production in a dose-dependent manner (Figures 1B, C). In addition, the W-DP remarkably decreased the melanin contents in the cell pellets, which showed lighter colors (Figure 1D). These results suggest that W-DP decreases melanin synthesis through the downregulation of tyrosinase.

\section{Effect of W-DP on the Expression of Melanogenesis-Related Proteins in $\alpha-$ MSH-Induced B16-F10 Cells}

We tested the inhibitory effect of W-DP on melanogenesisrelated proteins by Western blot analysis. The expression levels of MITF and tyrosinase were significantly reduced by treatment with W-DP in a dose-dependent manner. TRP-1 and TRP-2 stimulated by $\alpha-\mathrm{MSH}$ were also simultaneously decreased by $\mathrm{W}$ DP treatment (Figure 2). Our results suggest that its effect on melanin synthesis may be associated with the suppression of melanogenesis-related proteins such as tyrosinase, TRP-1, and TRP-2 through MITF inhibition.

\section{Structure Elucidation of DMW-1 and 2 in D. morbiferus}

To identify the anti-melanogenic active constituents, we isolated 22 components from the ethyl acetate fraction (W-EA), which was separated from $D$. morbiferus by continuous fractionation (see Supplementary Figure S1). The crude water extract of $D$. morbiferus leaves was subjected to a succession of fractionation procedures and the W-EA showed the most significant inhibitory effects. We evaluated the effects of W-EA at various concentrations in $\alpha$-MSH-activated B16-F10 cells. W-EA significantly inhibited tyrosinase activity in a dose-dependent 

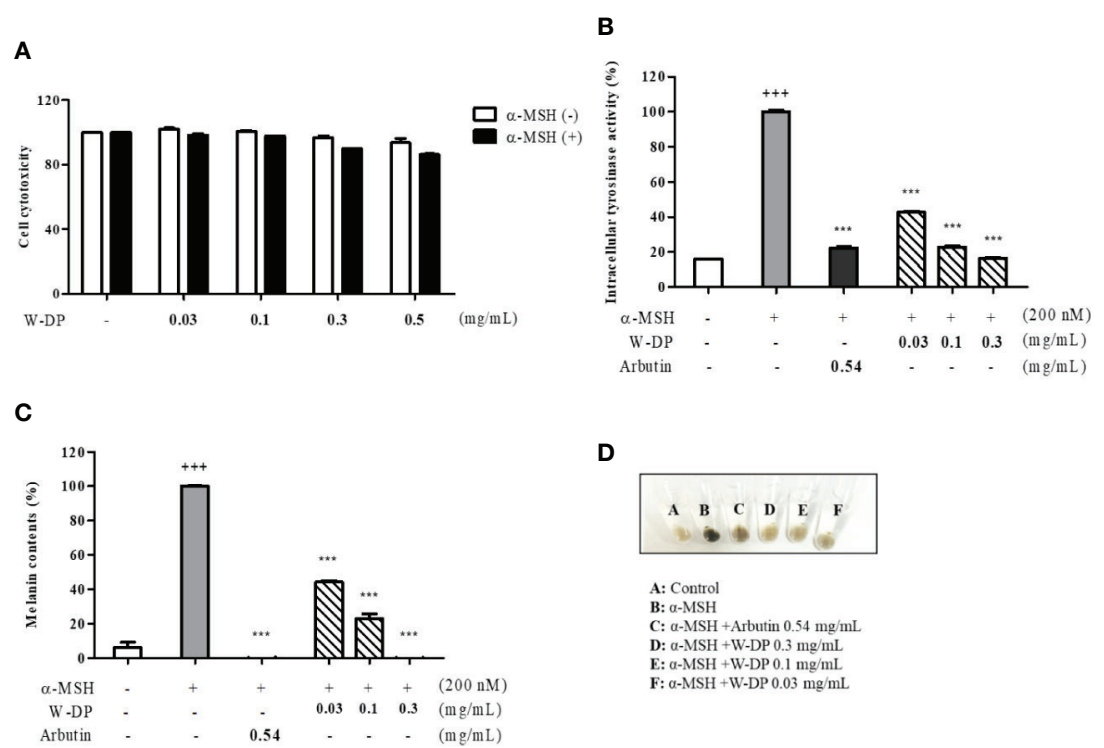

D

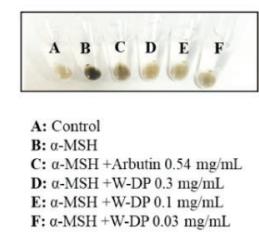

FIGURE 1 | Effect of W-DP on melanogenesis in $\alpha-M S H-i n d u c e d ~ B 16-F 10$ cells. B16-F10 cells pretreated with different concentrations of W-DP (0.03, 0.1, 0.3, 0.5 $\mathrm{mg} / \mathrm{ml})$ were incubated with or without $\alpha-\mathrm{MSH}(200 \mathrm{nM})$ for $72 \mathrm{~h}$. (A) Cell viability was tested by MTS. (B) The cells were then lysed and quantified by using Bradford

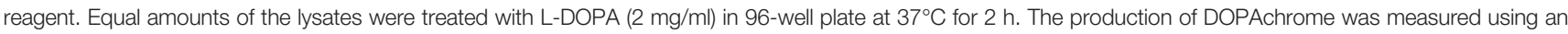
ELISA microplate reader at an absorbance of $490 \mathrm{~nm}$. (C) The cells were dissolved in $1 \mathrm{~N} \mathrm{NaOH}$ with $10 \%$ DMSO for $1 \mathrm{~h}$ at $60^{\circ} \mathrm{C}$. Total melanin lysates were transferred to a 96-well plate and the optical absorbance was measured at $405 \mathrm{~nm}$ using an ELISA microplate reader. (D) Colors of the pellets indicated the change of melanogenesis from $\alpha$-MSH-induced B16-F10 cells. Data are expressed as the mean \pm SEM of three independent experiments. ${ }^{* \star *} P<0.001$ versus $\alpha$-MSH stimulation. ${ }^{+++} P<0.001$ versus the untreated control.

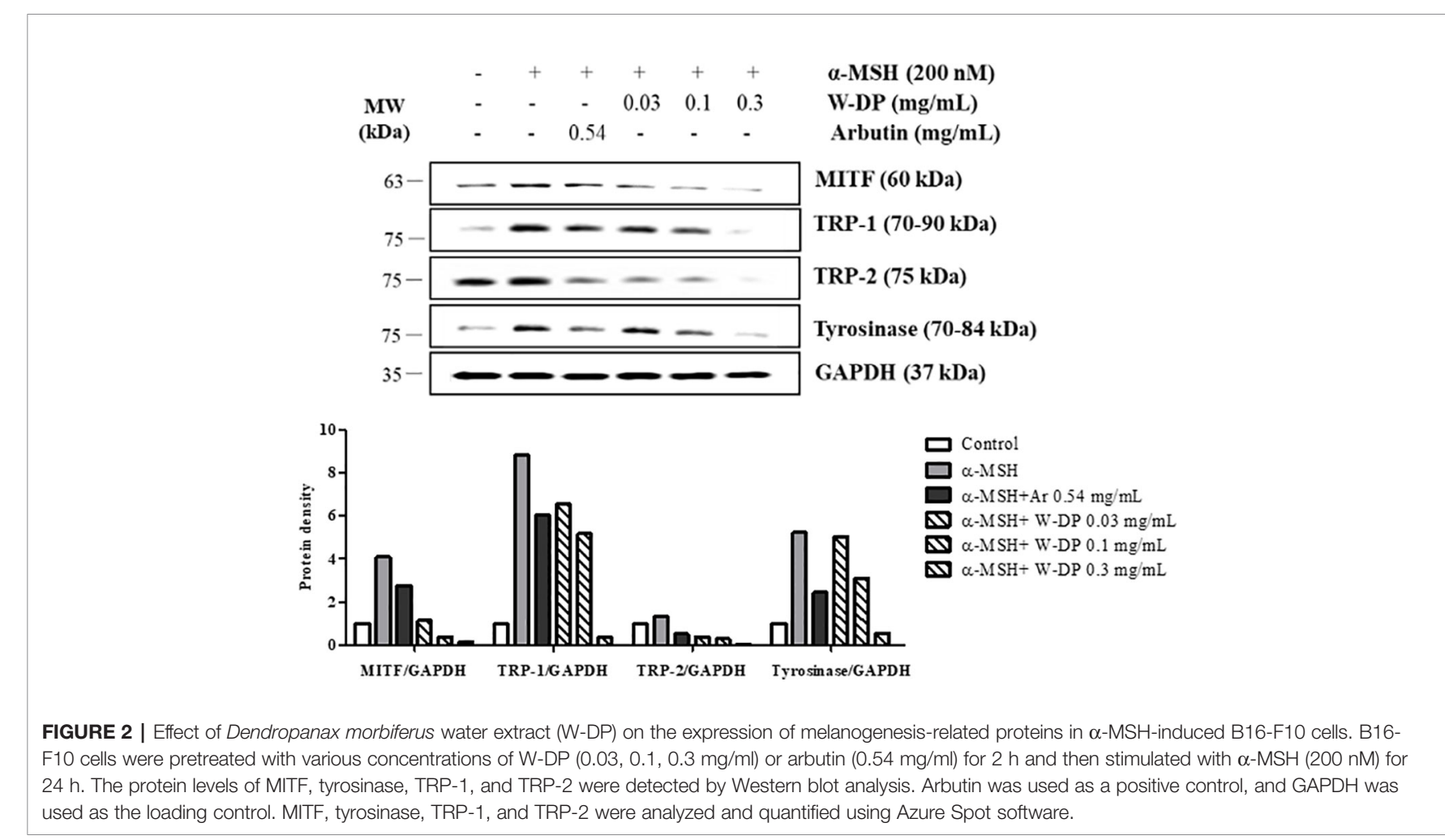


manner. W-EA at the concentrations of $\sim 0.001,0.01$, and 0.03 $\mathrm{mg} / \mathrm{ml}$ showed $45 \%, 53 \%$, and $65 \%$ inhibitory effects, respectively. W-EA was confirmed to be sufficiently effective even at a concentration of $0.01 \mathrm{mg} / \mathrm{ml}$ lower than $0.03 \mathrm{mg} / \mathrm{ml}$. We showed the one concentration of W-EA results to compare the efficacy of single ingredients (data not shown). Of all isolated components, (10E)-9,16-dihydroxyoctadeca-10,17-dien-12,14diynoate (DMW-1) and (10E)-(-)-10,17-octadecadiene-12,14diyne-1,9,16-triol (DMW-2) significantly suppressed melanin contents in $\alpha-\mathrm{MSH}$-induced B16-F10 cells (Supplementary Table S2). DMW-1 had a molecular formula of $\mathrm{C}_{19} \mathrm{H}_{26} \mathrm{O}_{4}$ by HRESIMS ([M+NH 4$]^{+} m / z 336.2167$, calcd for 336.2175) and obtained as a viscous liquid (see Supplementary Figure S2). The $1 \mathrm{H}-\mathrm{NMR}$ spectrum showed signals for five olefinic protons at $\delta$ $6.34(\mathrm{dd}, J=15.9,5.7 \mathrm{~Hz}), 5.94(\mathrm{ddd}, J=17.1,10.2,5.4 \mathrm{~Hz}), 5.79$ (ddd, $J=15.9,1.6,0.9 \mathrm{~Hz}), 5.42(\mathrm{~d}, J=17.1 \mathrm{~Hz}), 5.22(\mathrm{~d}, J=10.2$ $\mathrm{Hz})$ and $4.96(\mathrm{dd}, J=5.4,1.1 \mathrm{~Hz})$, two oxygenated methane protons at $\delta 4.96(\mathrm{dd}, J=5.4,1.1 \mathrm{~Hz})$ and $4.13(\mathrm{dtd}, J=7.2,5.7$, $1.6 \mathrm{~Hz}$ ), and one methoxy proton at $\delta 3.76(\mathrm{~s})$. The ${ }^{13} \mathrm{C}-\mathrm{NMR}$ spectrum revealed the presence of a carbonyl carbon at $\delta 176.0$, four olefinic carbons at $\delta 151.9,138.1,116.6$, and 108.4, four quaternary carbons at $\delta 82.2,78.0,74.0$, and 70.6, two oxygenbearing carbons at $\delta 72.4$ and 64.0, and one methoxy carbon at $\delta$ 52.0 (see Supplementary Figure S2 and Table S1). The above analyses suggested that DMW-1 should be a polyacetylene compound. The NMR results of DMW-1 were similar to those of the known DMW-2, except for a methoxy-carbonyl group (see Supplementary Figure S3 and Table S1). DMW-1 and DMW-2 have a negative optical rotation value $\left([\alpha]-38.0^{\circ}\right.$ and $\left.-32.6^{\circ}\right)$, indicating the same relative configuration. The structures of DMW-1 and 2 components were identified by NMR spectroscopy and are shown in Figure 3A. DMW-1 and 2 were quantified in W-EA by LC/MS/MS analysis. The DMW-2 content $(637 \mathrm{ng} / \mathrm{ml})$ was dramatically higher than the DMW-1 content $(87.1 \mathrm{ng} / \mathrm{ml})$ in the W-EA fraction (Figure 3B). The reaction times of DMW-1 and DMW-2 were 6.2 and 5.43 min, respectively.

\section{Inhibitory Effect of DMW-1 and 2 on Melanogenesis in $\alpha-M S H-I n d u c e d ~ B 16-F 10$ Cells}

To examine the effects of DMW-1 and 2 on melanogenesis, B16F10 cells were pretreated with two compounds and then stimulated with $\alpha$-MSH for 72 h. DMW-1 and 2 showed significant decreases in tyrosinase activity and melanin production. Notably, DMW-1 showed a better inhibitory effect than DMW-2 in B16-F10 cells. DMW-1 reduced the intracellular tyrosinase activity with an estimated $\mathrm{IC}_{50}$ of $1.175 \mu \mathrm{M}$ and decreased the melanin accumulation with an estimated $\mathrm{IC}_{50}$ of $1.221 \mu \mathrm{M}$. The inhibitory effects of DMW-1 approached the effect of the reference compound arbutin (Figures 4A, B). As shown in Figure 4C, both compounds resulted in decreased melanin contents with lighter colors. Based on the above results, we studied whether DMW-1 and 2 could inhibit melanogenesisrelated protein expression by Western blot analysis. DMW-1 and 2 remarkably decreased the protein expression levels of MITF, tyrosinase, and TRP-1 (Figure 4D). These results indicate that the anti-melanogenic effects of DMW-1 and 2 may be mediated by downregulating melanogenesis-related proteins.

\section{Effect of DMW-1 and 2 on the Phosphorylation Levels of PKA and CREB in $\alpha$-MSH-Induced B16-F10 Cells}

To investigate the effect of DMW-1 and 2 on cAMP-related signaling pathway, phosphorylation levels of PKA and CREB were analyzed by Western blot analysis. Compared to W-DP and $\mathrm{W}$-EA, the phosphorylation levels of PKA and CREB proteins were markedly reduced by the treatment of DMW-1 and 2 in $\alpha$ MSH-induced B16-F10 cells. However, PKA and CREB levels

\section{A}

DMW-1 (methyl (10E)-9,16-dihydroxyoctadeca-10,17-dien-12,14-diynoate) MW: 318.1831

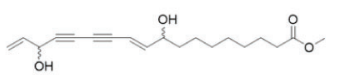

DMW-2 ((10E)-(-)-10,17-Octadecadiene-12,14-diyne-1,9,16-triol; Fruticotriol) MW: 290.1882

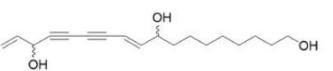

B

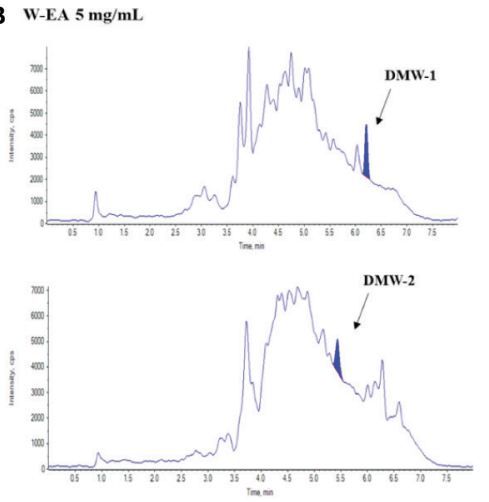

FIGURE 3 | Chemical structures and chromatograms of DMW-1 and 2. (A) Chemical structures of DMW-1 and 2 and (B) total ion chromatogram of DMW-1 and 2 in W-EA at $5 \mathrm{mg} / \mathrm{ml}$. W-EA, ethyl acetate fraction. 

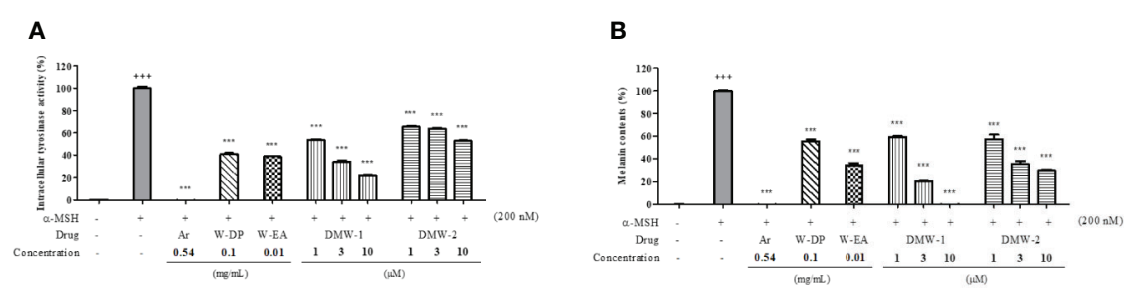

C
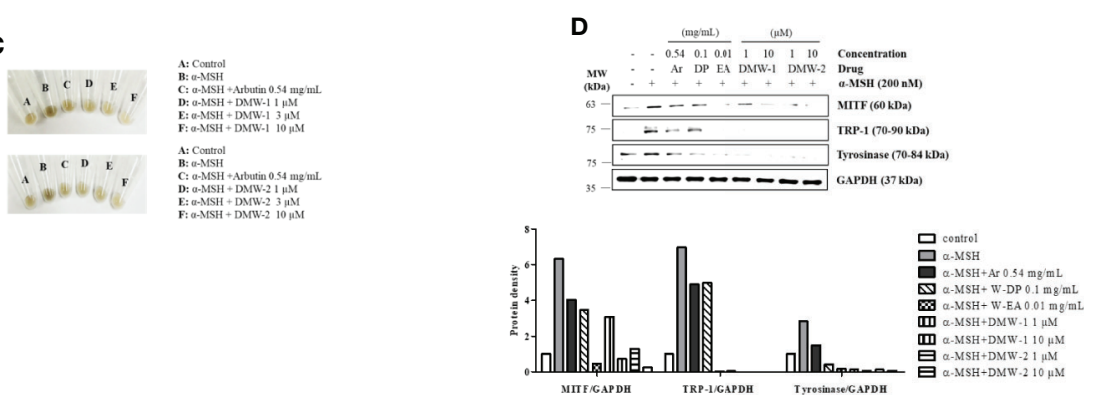

FIGURE 4 | Effects of DMW-1 and 2 on melanogenesis in $\alpha-M S H$-induced B16-F10 cells. B16-F10 cells $\left(1 \times 10^{5}\right.$ cells/well) were incubated into six-well plate at $37^{\circ} \mathrm{C}$ overnight. B16-F10 cells were pretreated with W-DP $(0.1 \mathrm{mg} / \mathrm{ml})$, W-EA $(0.01 \mathrm{mg} / \mathrm{ml})$, DMW-1 (1, 3, $\left.10 \mu \mathrm{M}\right)$, DMW-2 $(1,3,10 \mu \mathrm{M})$, or arbutin $(0.54 \mathrm{mg} / \mathrm{ml})$ for $2 \mathrm{~h}$ prior to stimulation with $\alpha-\mathrm{MSH}(200 \mathrm{nM})$ for $72 \mathrm{~h}$. (A) Equal amounts of the lysates were treated with L-DOPA $(2 \mathrm{mg} / \mathrm{ml})$ at $37^{\circ} \mathrm{C}$ for $2 \mathrm{~h}$. The production of DOPAchrome was measured at an absorbance of $490 \mathrm{~nm}$. (B) The cells were dissolved in $1 \mathrm{~N} \mathrm{NaOH}$ with $10 \% \mathrm{DMSO}$ for $1 \mathrm{~h}$ at $60^{\circ} \mathrm{C}$. Total melanin lysates were measured at an absorbance of $405 \mathrm{~nm}$. (C) Colors of the pellets indicated the change of melanogenesis from $\alpha-M S H$-induced B16-F10 cells. (D) The expression levels of MITF, tyrosinase, and TRP-1 were determined by Western blot analysis, and the protein bands were quantified using Azure Spot software. GAPDH was used as the loading control. Data are expressed as the mean \pm SEM of three independent experiments. ${ }^{* * *} P<0.001$ versus $\alpha$-MSH stimulation. ${ }^{+++} P<0.001$ versus the untreated control. Ar, arbutin; W-DP, D. morbiferus water extract; W-EA, ethyl acetate fraction.

remained unchanged. DMW-1 showed a slightly greater inhibitory effect on the phosphorylation levels of PKA/CREB than DMW-2 in B16-F10 cells (Figure 5A). Moreover, the cAMP level accumulated in the lysates was evaluated using competitive ELISA. As shown in Figure 5B, DMW-1 decreased intracellular cAMP levels in a dose-dependent manner.

\section{Effect of DMW-1 and 2 on the Phosphorylation of p38 in $\alpha$-MSH- Stimulated B16-F10 Cells}

To further investigate whether DMW-1 and 2 exert antimelanogenic effect through regulating the MAPK pathway, the phosphorylation levels of MAPKs were assayed by Western blot
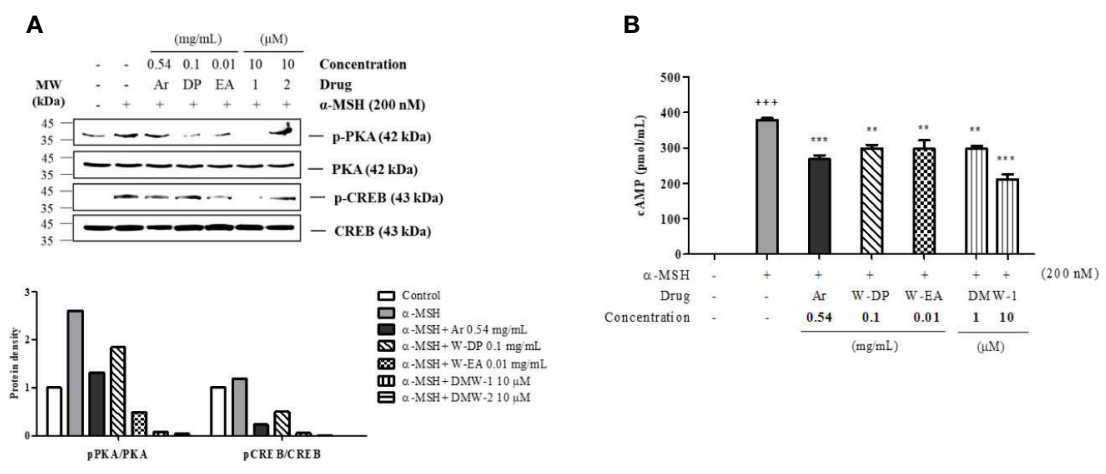

FIGURE 5 | Effects of DMW-1 and 2 on the phosphorylation levels of PKA and CREB in $\alpha$-MSH-induced B16-F10 cells. The cells were pretreated with W-DP (0.1 $\mathrm{mg} / \mathrm{ml}), \mathrm{W}$-EA $(0.01 \mathrm{mg} / \mathrm{ml})$, DMW-1 $(10 \mu \mathrm{M})$, DMW-2 $(10 \mu \mathrm{M})$, or arbutin $(0.54 \mathrm{mg} / \mathrm{ml})$ for $2 \mathrm{~h}$ before stimulation with $\alpha-\mathrm{MSH}(200 \mathrm{nM})$ for $24 \mathrm{~h}$. (A) The p-PKA and p-CREB protein levels were determined by Western blot analysis, and normalized to total PKA and CREB. The phosphorylation levels of PKA and CREB were analyzed and quantified using Azure Spot software. (B) The lysates were collected and cAMP levels were tested by ELISA. Data are expressed as the mean \pm SEM of three independent experiments. ${ }^{* *} P<0.01$, and ${ }^{* * *} P<0.001$ versus $\alpha-\mathrm{MSH}$ stimulation. ${ }^{+++} P<0.001$ versus the untreated control. Ar, arbutin; W-DP, $D$. morbiferus water extract; W-EA, ethyl acetate fraction. 

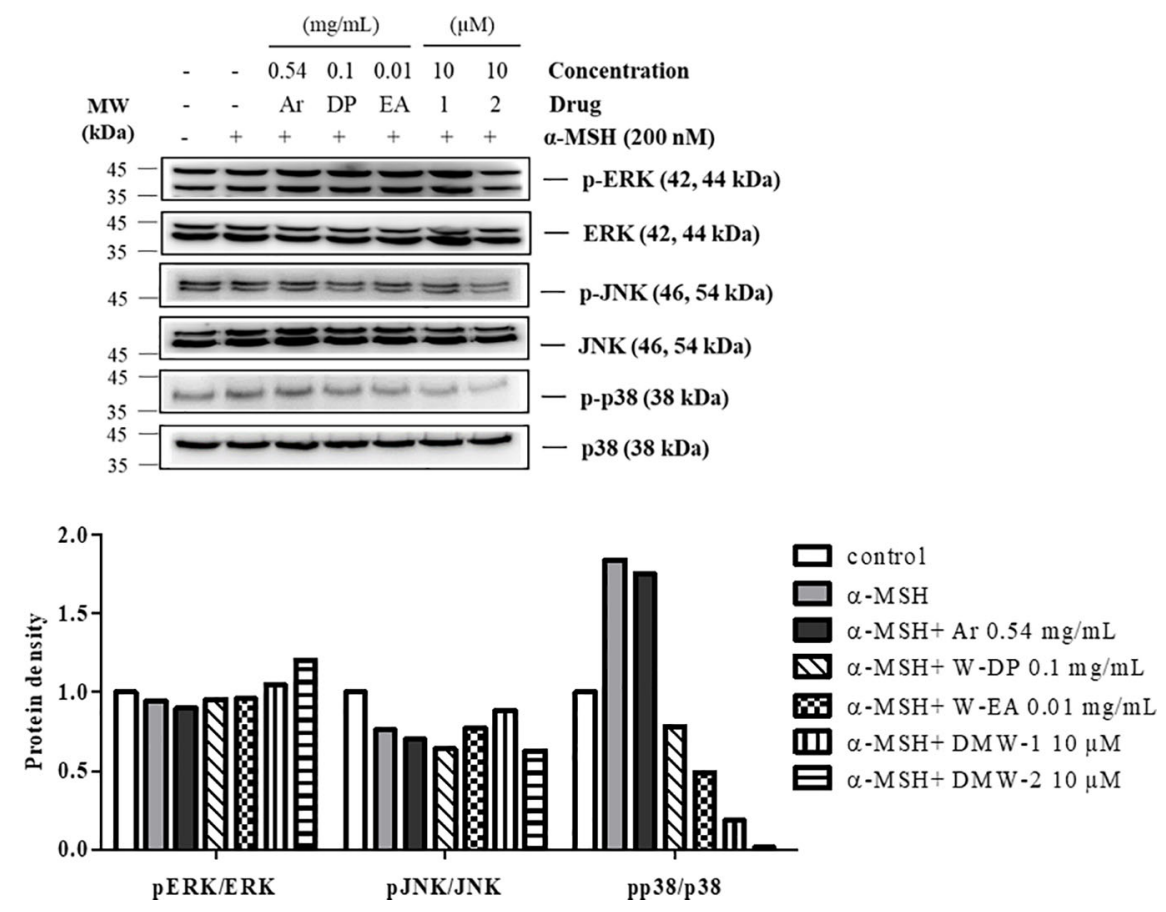

FIGURE 6 | Effect of DMW-1 and 2 on p38 phosphorylation in $\alpha-M S H$-stimulated B16-F10 cells. B16-F10 cells were pretreated with W-DP (0.1 mg/ml), W-EA (0.01 $\mathrm{mg} / \mathrm{ml})$, DMW-1 $(10 \mu \mathrm{M})$, DMW-2 $(10 \mu \mathrm{M})$, or arbutin $(0.54 \mathrm{mg} / \mathrm{ml})$ for $2 \mathrm{~h}$ before stimulation with $\alpha-\mathrm{MSH}(200 \mathrm{nM})$ for $24 \mathrm{~h}$. Phosphorylation levels of ERK, JNK, and p38 were determined by Western blot analysis. Equal levels of proteins were confirmed using total ERK, JNK, and p38 antibodies. The protein bands were analyzed and quantified using Azure Spot software. Ar, arbutin; W-DP, D. morbiferus water extract; W-EA, ethyl acetate fraction.

analysis. As shown in Figure 6, DMW-1 and 2 significantly decreased p38 phosphorylation. In contrast, the phosphorylation levels of ERK and JNK proteins showed no increase after treatment with DMW-1 and 2 in B16-F10 cells. These data indicate that the anti-melanogenic activity of DMW-1 is associated with the decrease of p-p38 as well as p-CREB.

\section{DISCUSSION}

There is an increasing interest in medicinal plants and their bioactive compounds for novel whitening therapies in recent years. Various cosmetic ingredients such as arbutin, kojic acid, and vitamin $\mathrm{C}$ have been used to inhibit hyperpigmentation $(\mathrm{Ng}$

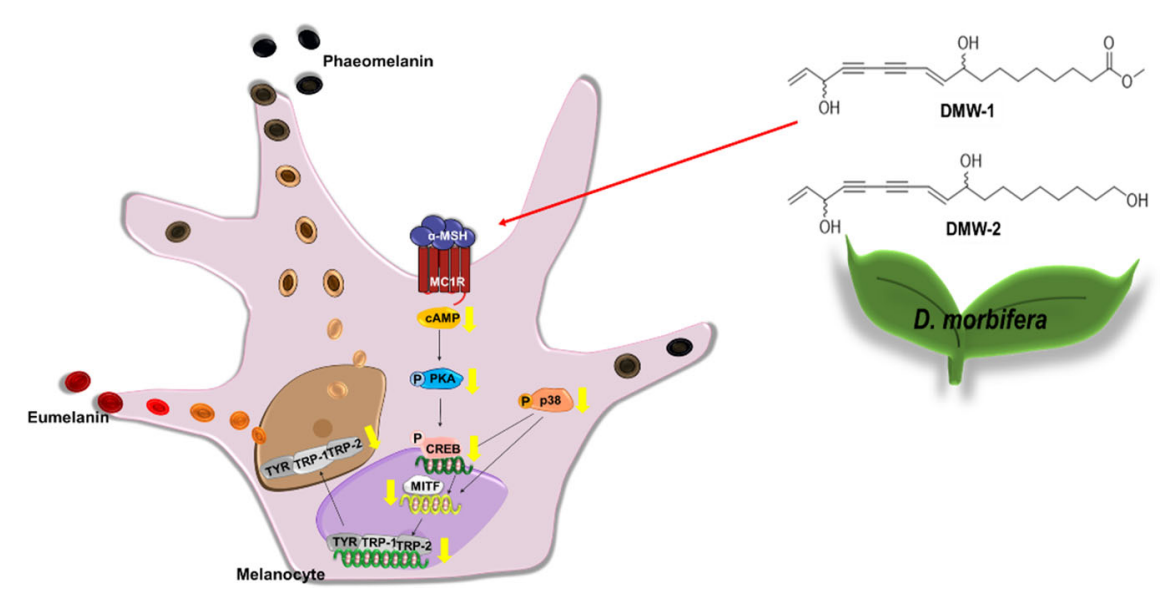

FIGURE 7 | Underlying potential mechanism of anti-melanogenesis of $D$. morbiferus. The anti-melanogenesis effect of $D$. morbiferus might be associated with downregulation of MITF. D. morbiferus and its active component DMW-1 significantly inhibited melanin formation by inhibition of melanogenesis-related proteins such as tyrosinase, TRP-1, and TRP-2. D. morbiferus and DMW-1 show multifunctional inhibitory activities on melanogenesis pathways via PKA/CREB- and p38 MAPKmediated MITF degradation. 
et al., 2014). We have studied to identify herbal medicines showing anti-melanogenic effects without cytotoxicity. To the best of our knowledge, this is the first report to demonstrate the anti-melanogenic effect of $D$. morbiferus leaves and its new single constituents. In this study, we investigated the underlying mechanisms of D. morbiferus -mediated anti-melanogenic effects and identified the active compounds. Interestingly, $D$. morbiferus significantly decreased melanin content in $\alpha-\mathrm{MSH}-$ induced B16-F10 cells (Figure 1). D. morbiferus did not show any cytotoxicity to B16-F10 cells (Figure 1A). Melanogenesis regulation is under complex control. Melanin synthesis can be regulated by the direct suppression of tyrosinase and by the downregulation of tyrosinase or other melanogenesis-related proteins. Here, we have investigated the molecular mechanisms of D. morbiferus on anti-melanogenic effects focusing on tyrosinase, TRP-1, TRP-2, MITF, cAMP, PKA/CREB, and p38 MAPK pathways. Interestingly, D. morbiferus markedly reduced the expression levels of MITF, TRP-1, TRP-2, and tyrosinase in a dose-dependent manner (Figure 2). Next, we isolated 22 components from ethyl acetate fraction of $D$. morbiferus. Of all the isolated components, DMW-1 and 2 significantly inhibited melanin contents in $\alpha-\mathrm{MSH}$-induced B16-F10 cells (Supplementary Table S2). DMW-1 and 2 are polyacetylenebased compounds with similar structures (Figure 3), and their structural explanations are provided in the supplementary data (Supplementary Figures S2 and S3 and Table S1). Of all polyacetylene-based compounds, several studies have reported that (9Z,16S)-16-hydroxy-9,17-octadecadiene-12,14-diynoic acid has anti-obesity, anti-osteoclastogenic, antioxidant, antiinflammatory, and anticomplement activities (Park et al., 2004; Kang et al., 2018; Kim et al., 2018a). The beneficial effect of (2Z, $8 Z$ )-matricaria acid methyl ester has been reported to inhibit tyrosinase activity (Luo et al., 2009). In this study, we confirmed that DMW-1 and 2 dramatically suppressed tyrosinase activity and melanin overproduction. Consistently, the expression levels of MITF, tyrosinase, and TRP-1 were remarkably decreased by DMW-1 and 2 (Figure 4). We found that DMW-1 and 2 dramatically inhibited melanogenesis through the downregulation of MITF and tyrosinase. DMW-1 showed significant inhibitory effect on melanogenesis, which indicated that the active compound was abundant in D. morbiferus. CREB regulates the expression of MITF in melanosomes and the phosphorylation of CREB is regulated by the activation of cAMP/PKA cascades that are known to play main roles in melanin synthesis (Pillaiyar et al., 2017). The inhibitory effect of DMW-1 on the phosphorylation levels of PKA and CREB was slightly greater than DMW-2 in B16-F10 cells, and DMW-1 significantly decreased intracellular cAMP levels in a dose- dependent manner (Figure 5). Next, we examined the role of the MAPK signaling pathway in the effects of two chemical constituents on melanogenesis. DMW-1 and 2 significantly decreased p38 phosphorylation in B16-F10 cells. In contrast, the phosphorylation levels of ERK and JNK were not increased by the treatment of DMW-1 and 2 in B16-F10 cells (Figure 6). These results suggested that the PKA/CREB and p38-mediated signaling pathways were involved in melanin production and tyrosinase activity, which were affected by DMW-1. These results indicate that DMW-1 isolated from D. morbiferus has antimelanogenic effect via PKA/CREB- and p38 MAPK-mediated MITF degradation.

In conclusion, D. morbiferus and its active component DMW-1 significantly inhibited melanin formation by inhibition of melanogenesis-related proteins such as tyrosinase, TRP-1, and TRP-2, without any cytotoxicity. D. morbiferus and DMW-1 show multifunctional inhibitory activities on melanogenesis pathways via PKA/CREB- and p38 MAPKmediated MITF degradation (Figure 7). These results suggested that D. morbiferus and DMW-1 might be potential ingredients for application in cosmetics and therapeutic agents for skin hyperpigmentation disorders.

\section{DATA AVAILABILITY STATEMENT}

The datasets generated for this study are available on request to the corresponding authors.

\section{AUTHOR CONTRIBUTIONS}

JP was involved in the project design, carried out major experiments, and drafted the manuscript. SY and HL participated to extract the active compounds from $D$. morbiferus and reviewed the protocol. RG wrote and revised the manuscript. YRK and YHK conceived the project, provided reagents, and conceptual design, and wrote the manuscript. All authors read and approved the manuscript finally.

\section{SUPPLEMENTARY MATERIAL}

The Supplementary Material for this article can be found online at: https://www.frontiersin.org/articles/10.3389/ fphar.2020.00507/full\#supplementary-material

\section{REFERENCES}

Birhanu, B. T., Kim, J. Y., Hossain, M. A., Choi, J. W., Lee, S. P., and Park, S. C. (2018). An in vivo immunomodulatory and anti-inflammatory study of fermented Dendropanax morbifera Leveille leaf extract. BMC Complement Altern. Med. 18, 1-8. doi: 10.1186/s12906-018-2282-x

Brenner, M., and Hearing, V. J. (2008). The protective role of melanin against UV damage in human skin. Photochem. Photobiol. 84 (3), 539-549. doi: 10.1111/ j.1751-1097.2007.00226.x

Chan, C. F., Huang, C. C., Lee, M. Y., and Lin, Y. S. (2014). Fermented broth in tyrosinase- and melanogenesis inhibition. Molecules 19 (9), 13122-13135. doi: $10.3390 /$ molecules190913122 
Cho, S. S., Song, S. H., Choi, C. Y., Park, K. M., Shim, J. H., and Park, D. H. (2018). Optimization of the extraction conditions and biological evaluation of Dendropanax morbifera $\mathrm{H}$. Lev as an anti-hyperuricemic source. Molecules 23 (12), 3313-3320. doi: 10.3390/molecules23123313

Choo, G. S., Lim, D. P., Kim, S. M., Yoo, E. S., Kim, S. H., Kim, C. H., et al. (2019). Anti-inflammatory effects of Dendropanax morbifera in lipopolysaccharide -stimulated RAW264.7 macrophages and in an animal model of atopic dermatitis. Mol. Med. Rep. 19, 2087-2096. doi: 10.3892/mmr.2019.9887

Costin, G. E., and Hearing, V. J. (2007). Human skin pigmentation: melanocytes modulate skin color in response to stress. FASEB J. 21 (4), 976-994. doi: 10.1096/fj.06-6649rev

D'Orazio, J., Jarrett, S., Amaro-Ortiz, A., and Scott, T. (2013). UV radiation and the skin. Int. J. Mol. Sci. 14 (6), 12222-12248. doi: 10.3390/ijms140612222

Deng, J. X., Kim, C. S., Oh, E. S., and Yu, S. H. (2010). First report of foliar blight on dendropanax morbifera caused by alternaria panax. Mycobiology 38 (4), 316-320. doi: 10.4489/MYCO.2010.38.4.316

Jeong, B. S., Jo, J. S., Pyeo, B. S., and Hwang, B. (1995). Studies on the distribution of Dendropanax morbifera and component analysis of the golden lacquer. Korean J. Biotechnol. Bioeng. 10 (4), 393-400.

Kang, M. J., Kwon, E. B., Ryu, H. W., Lee, S., Lee, J. W., Kim, D. Y., et al. (2018). Polyacetylene from Dendropanax morbifera alleviates diet-induced obesity and hepatic steatosis by activating AMPK signaling pathway. Front. Pharmacol. 9, 537-547. doi: 10.3389/fphar.2018.00537

Kim, E. H., Jo, C. S., Ryu, S. Y., Kim, S. H., and Lee, J. Y. (2018a). Antiosteoclastogenic diacetylenic components of Dendropanax morbifera. Arch. Pharm. Res. 41 (5), 506-512. doi: 10.1007/s12272-018-1033-3

Kim, J. Y., Lee, E. J., Ahn, Y., Park, S., Kim, S. H., and Oh, S. H. (2018b). A chemical compound from fruit extract of Juglans mandshurica inhibits melanogenesis through p-ERK-associated MITF degradation. Phytomedicine 57, 57-64. doi: 10.1016/j.phymed.2018.12.007

Lee, B. H., Choi, J. H., and Kim, H. J. (2005). Curing and thermal behaviors of Korean Dendropanax Lacquer made by acetone and wine spirit extraction methods. Prog. Org. Coatings 52 (3), 241-245. doi: 10.1016/ j.porgcoat.2005.01.002

Lee, J. W., Kim, K. S., An, H. K., Kim, C. H., Moon, H. I., and Lee, Y. C. (2013). Dendropanoxide induces autophagy through ERK1/2 activation in MG-63 human osteosarcoma cells and autophagy inhibition enhances dendropanoxide-induced apoptosis. PloS One 8 (12), 83611-83617. doi: 10.1371/journal.pone.0083611

Lee, S. Y., Choi, E. J., Bae, D. H., Lee, D. W., and Kim, S. O. (2015). Effects of 1tetradecanol and $\beta$-sitosterol Isolated from Dendropanax morbifera Lev. OnSkin Whitening, Moisturizing and Preventing Hair Loss. J. Soc Cosmet. Sci. Korea 41, 73-83. doi: 10.15230/SCSK.2015.41.1.73

Li, H. X., Park, J. U., Su, X. D., Kim, K. T., Kang, J. S., Kim, Y. R., et al. (2018). Identification of anti-melanogenesis constituents from Morus alba L. Leaves. Molecules 23 (10), 2559-2569. doi: 10.3390/molecules23102559

Lim, L., Yun, J. J., Jeong, J. E., Wi, A. J., and Song, H. (2015). Inhibitory effects of nano-extract from Dendropanax morbifera on proliferation and migration of vascular smooth muscle cells. J. Nanosci. Nanotechnol. 15 (1), 116-119. doi: 10.1166/jnn.2015.8382

Liu, J. J., and Fisher, D. E. (2010). Lighting a path to pigmentation: mechanisms of MITF induction by UV. Pigment. Cell. Melanoma. Res. 23 (6), 741-745. doi: 10.1111/j.1755-148X.2010.00775.x

Luo, L. H., Kim, H. J., Nguyen, D. H., Lee, H. B., Lee, N. H., and Kim, E. K. (2009). Depigmentation of melanocytes by $(2 \mathrm{Z}, 8 \mathrm{Z})$-matricaria acid methyl ester isolated from Erigeron breviscapus. Biol. Pharm. Bull. 32 (6), 1091-1094. doi: 10.1248/bpb.32.1091

Ng, L. T., Lin, L. T., Chen, C. L., Chen, H. W., Wu, S. J., and Lin, C. C. (2014). Antimelanogenic effects of delta-tocotrienol are associated with tyrosinase-related proteins and MAPK signaling pathway in B16 melanoma cells. Phytomedicine 21 (7), 978-983. doi: 10.1016/j.phymed.2014.03.003

Park, Y. M., and Han, J. S. (2016). A study on the utilization of Dendropanax morbifera Lev. leaf extract for material of functional cosmetics and hair growth products. Asian J. Beauty Cosmetol. 14 (3), 277-288. doi: 10.20402/ ajbc.2016.0051

Park, B. Y., Min, B. S., Oh, S. R., Kim, J. H., Kim, T. J., Kim, D. H., et al. (2004). Isolation and anticomplement activity of compounds from Dendropanax morbifera. J. Ethnopharmacol. 90 (2-3), 403-408. doi: 10.1016/ j.jep.2003.11.002

Park, S. E., Sapkota, K., Choi, J. H., Kim, M. K., Kim, Y. H., Kim, K. M., et al. (2014). Rutin from Dendropanax morbifera leveille protects human dopaminergic cells against rotenone induced cell injury through inhibiting JNK and p38 MAPK signaling. Neurochem. Res. 39 (4), 707-718. doi: 10.1007/ s11064-014-1259-5

Park, J. U., Kang, B. Y., Lee, H. J., Kim, S., Bae, D., Park, J. H., et al. (2017). Tetradecanol reduces EL-4 T cell growth by the down regulation of NF-kappaB mediated IL-2 secretion. Eur. J. Pharmacol. 799, 135-142. doi: 10.1016/ j.ejphar.2017.02.002

Park, J. U., Kang, B. Y., and Kim, Y. R. (2018a). Ethyl acetate fraction from Dendropanax morbifera leaves Iicreases T cell growth by upregulating NF-ATmediated IL-2 secretion. Am. J. Chin. Med. 46 (2), 453-467. doi: 10.1142/ S0192415X18500234

Park, S. Y., Karthivashan, G., Ko, H. M., Cho, D. Y., Kim, J., Cho, D. J., et al. (2018b). Aqueous extract of Dendropanax morbiferus leaves effectively alleviated neuroinflammation and behavioral impediments in MPTPinduced parkinson's mouse model. Oxid. Med. Cell. Longev. 2018, 31752143175228. doi: 10.1155/2018/3175214

Pillaiyar, T., Manickam, M., and Jung, S. H. (2017). Downregulation of melanogenesis: drug discovery and therapeutic options. Drug Discovery Today 22 (2), 282-298. doi: 10.1016/j.drudis.2016.09.016

Pillaiyar, T., Namasivayam, V., Manickam, M., and Jung, S. H. (2018). Inhibitors of melanogenesis: an updated review. J. Med. Chem. 61 (17), 7395-7418. doi: 10.1021/acs.jmedchem.7b00967

Riley, P. A. (2003). Melanogenesis and melanoma. Pigment. Cell. Res. 16 (5), $548-$ 552. doi: 10.1034/j.1600-0749.2003.00069.x

Slominski, A., Moellmann, G., Kuklinska, E., Bomirski, A., and Pawelek, J. (1988). Positive regulation of melanin pigmentation by two key substrates of the melanogenic pathway, L-tyrosine and L-dopa. J. Cell. Sci. 89, 287-296.

Slominski, A., Zmijewski, M. A., and Pawelek, J. (2012). L-tyrosine and Ldihydroxyphenylalanine as hormone-like regulators of melanocyte functions. Pigment. Cell. Melanoma. Res. 25 (1), 14-27. doi: 10.1111/j.1755148X.2011.00898.x

Song, J. H., Kang, H. B., Kim, J. H., Kwak, S., Sung, G. J., Park, S. H., et al. (2018). Antiobesity and cholesterol-lowering effects of Dendropanax morbifera water extracts in mouse 3T3-L1 Cells. J. Med. Food. 21 (8), 793-800. doi: 10.1089/ jmf.2017.4154

Sun, S., Kee, H. J., Jin, L., Ryu, Y., Choi, S. Y., Kim, G. R., et al. (2018a). Gentisic acid attenuates pressure overload-induced cardiac hypertrophy and fibrosis in mice through inhibition of the ERK1/2 pathway. J. Cell. Mol. Med. 22 (12), 5964-5977. doi: 10.1111/jcmm.13869

Sun, S., Li, T., Jin, L., Piao, Z. H., Liu, B., Ryu, Y., et al. (2018b). Dendropanax morbifera prevents cardiomyocyte hypertrophy by inhibiting the Sp1/GATA4 pathway. Am. J. Chin. Med. 46 (5), 1021-1044. doi: 10.1142/ S0192415X18500532

Wu, L. C., Lin, Y. Y., Yang, S. Y., Weng, Y. T., and Tsai, Y. T. (2011). Antimelanogenic effect of c-phycocyanin through modulation of tyrosinase expression by upregulation of ERK and downregulation of p38 MAPK signaling pathways. J. Biomed. Sci. 18, 74-84. doi: 10.1186/1423-0127-18-74

Yu, H. Y., Kim, K. S., Lee, Y. C., Moon, H. I., and Lee, J. H. (2012). Oleifolioside A, a new active compound, attenuates LPS-stimulated iNOS and COX-2 expression through the downregulation of NF-kappa B and MAPK activities in RAW 264.7 macrophages. Evid. Based. Complement Alternat. Med. 2012, 18. doi: $10.1155 / 2012 / 637512$

Conflict of Interest: The authors declare that the research was conducted in the absence of any commercial or financial relationships that could be construed as a potential conflict of interest.

Copyright (C) 2020 Park, Yang, Guo, Li, Kim and Kim. This is an open-access article distributed under the terms of the Creative Commons Attribution License (CC BY). The use, distribution or reproduction in other forums is permitted, provided the original author(s) and the copyright owner(s) are credited and that the original publication in this journal is cited, in accordance with accepted academic practice. No use, distribution or reproduction is permitted which does not comply with these terms. 